\title{
Prevalence of Pelvic Vein Pathology in Patients with Cryptogenic Stroke and Patent Foramen Ovale Undergoing MRV Pelvis
}

\author{
Marcey Osgood ${ }^{\mathrm{a}}$ Elizabeth Budman ${ }^{\mathrm{a}}$ Raphael Carandang ${ }^{\mathrm{a}-\mathrm{c}}$ \\ Richard P. Goddeau, Jr. ${ }^{a}$ Nils Henninger ${ }^{a}$ d \\ Department of ${ }^{a}$ Neurology, ${ }^{b}$ Anesthesiology (Critical Care), ${ }^{c}$ Surgery, and ${ }^{d}$ Psychiatry, University of \\ Massachusetts Medical School, Worcester, Mass., USA
}

\section{Key Words}

Cryptogenic stroke - Patent foramen ovale - Deep venous thrombosis - Magnetic resonance venography

\begin{abstract}
Background: A substantial proportion of ischemic strokes has no any identified underlying cause. Notably, the prevalence of a patent foramen ovale (PFO) is increased in cryptogenic stroke (CS) populations, which may serve as a conduit for paradoxical emboli originating from deep vein thrombosis (DVT) including the pelvic veins. Yet, there are no published guidelines for the assessment of pelvic veins as part of the stroke workup and few studies have systematically investigated pelvic veins as a potential source for paradoxical emboli in CS patients. Further, there is a relative paucity of data regarding pelvic DVT in CS and results have been conflicting. Hence, we sought to determine the prevalence of pelvic DVT in select CS patients with PFO who underwent magnetic resonance venography (MRV). Methods: We retrospectively identified patients $(n=50)$ who underwent contrast-enhanced pelvic MRV at the discretion of the treating physician for the evaluation of CS in the presence of a PFO during hospitalization at a single academic stroke center between January 2011 through December 2013. Multivariable logistic regression analyses were used to assess for factors independently associated with the presence of an abnormal
\end{abstract}

MRV pelvis. Results: Patients ( $47 \pm 13$ years of age) had MRV performed $4 \pm 3$ days after their incident stroke. Nine patients had an abnormal MRV (18\%). Of these, four (8\%) had pelvic vein thrombosis and $5(10 \%)$ a May-Thurner anatomic variant. All patients with pelvic DVT were subsequently anticoagulated with warfarin (none had abnormal hypercoagulability testing). Clinical clues suggesting paradoxical embolism were present in as many as $40 \%$ of patients. On multivariable logistic regression, a history of any risk factors predisposing to DVT (OR 6.7; coefficient 1.9; BCa 95\% Cl $0.08-20.2 ; p=0.014)$ as well as the number of predisposing risk factors (OR 3.9; coefficient 1.4; $\mathrm{BCa} 95 \% \mathrm{Cl} 0.25-4.2 ; \mathrm{p}=$ $0.005)$ predicted the presence of pelvic vein pathology on MRV. Conclusion: We demonstrate a relatively high prevalence of pelvic DVT among select CS patients emphasizing the importance of considering the pelvic veins as a potential source for emboli particularly in the presence of risk factors known to predispose DVT. Because patients were included at the treating physician's discretion, our results reflect 'reallife' practice. Our results may be of clinical importance as inclusion of pelvic vein imaging in CS patients with PFO had impactful therapeutic and nosologic implications. Further study is needed to define patients most likely to benefit from pelvic vein imaging.

(c) 2015 S. Karger AG, Basel

\section{KARGER 125}

() 2015 S. Karger AG, Base

$1015-9770 / 15 / 0394-0216 \$ 39.50 / 0$

E-Mail karger@karger.com

www.karger.com/ced
Nils Henninger, MD

Departments of Neurology and Psychiatry

University of Massachusetts Medical School, 55 Lake Ave, North

Worcester, MA 01655 (USA)

E-Mail nils.henninger@umassmed.edu 


\section{Introduction}

A significant proportion of all ischemic strokes (26$40 \%$ ) has no identifiable underlying cause and is deemed cryptogenic in origin [1]. This rate may be as high as $64 \%$ among patients under the age of 55 [2]. Notably, these young cryptogenic stroke (CS) patients have an increased prevalence of patent foramen ovale (PFO) [2] as well as neuroimaging characteristics suggestive of an embolic source for their stroke $[1,3,4]$. Diagnostic workup for CS patients with $\mathrm{PFO}$ is variable and frequently includes lower extremity Doppler ultrasound studies (DUS) to exclude deep venous thrombosis (DVT) as a potential source for paradoxical emboli. However, the diagnostic yield varies widely with a reported prevalence of lower extremity DVT ranging from 0 to 57\% [5-9]. It has been suggested that paradoxical emboli originating from the pelvis may be an important alternate source of stroke in this population $[6,10-12]$. Yet, few studies have investigated the association between DVT and CS [5-9] and even less is known regarding the potential contribution of pelvic vein pathology as a source for paradoxical emboli in CS patients [10-12].

To gain further insight into this issue, we retrospectively determined the prevalence of pelvic vein pathology as a potential source for paradoxical emboli in CS patients with PFO and right to left shunt as well as negative lower extremity DUS who had magnetic resonance venography (MRV).

\section{Methods}

\section{Study Population}

This study was reviewed and approved by our Institutional Review Board. We retrospectively identified all patients who underwent contrast-enhanced pelvic MRV at the discretion of the treating physician for the evaluation of CS in the presence of a PFO. They were admitted to a single academic center between January of 2011 and December 2013. We adhered to the STrengthening the Reporting of OBservational studies in Epidemiology (STROBE) guidelines (www.strobe-statement.org).

Patient demographics, National Institutes of Health Stroke Scale (NIHSS) score, laboratory data, comorbidities, and pre-admission medications (including active alcohol and illicit drug use) were collected on all included patients $(n=50)$. In addition, we reviewed known thrombotic risk factors including previous history of thrombosis, cancer, hormone replacement therapy, oral contraceptives, prolonged travel, or history of $>1$ miscarriages. Lastly, we investigated for the presence of dyspnea or a Valsalva maneuver at the time of onset of stroke, waking up with stroke, sleep apnea, or migraine, which may serve as clues for paradoxical embolism [13]. All patients were classified as having CS after completion of diagnostic workup [14]. Specifically, all patients had negative or normal: cerebrovascular imaging (computed tomography angiography (CTA) or magnetic resonance angiography (MRA) of the head and neck), transthoracic echocardiogram (TTE) with injected agitated saline contrast (except for the presence of a $\mathrm{PFO}$ ); with $\mathrm{n}=13$ patients additionally undergoing transesophageal echocardiography due to poor image quality of their TTE, lower extremity DUS, laboratory data (including complete blood count and basic metabolic panel (sodium, potassium, chloride, blood urea nitrogen, creatinine, glucose, and calcium), and cholesterol panel) as well as the absence of atrial fibrillation on routine ECG and in-patient telemetry monitoring. Further, we calculated the Risk of Paradoxical Embolism Study (RoPE) score. This score represents an index that may help stratify patients according to the probability that a discovered PFO is incidental or stroke-related. It is derived from routinely available clinical and radiologic variables (age, comorbidities, stroke location) and ranges from 0 (likely incidental) to 10 (likely related) [15]. Finally, clinical charts were reviewed to collect data on outpatient 30-day event monitoring and hypercoagulability testing.

\section{MRV Protocol and Image Analysis}

Images were acquired on a 1.5-Tesla whole-body MRI scanner (GE Signa; GE Medical Systems, Milwaukee, Wisc., USA). Imaging sequences included: coronal T1, coronal fast multiplanar inversion-recovery (FMPIR), axial proton density fat-saturation, axial 2-D time-of-flight angiography, axial liver acquisition with volume acquisition (LAVA) pre-contrast, axial and coronal LAVA post-contrast, and multiple post-contrast coronal time-resolved imaging of contrast kinetics (TRICKS). All post-contrast images were obtained after intravenous administration of $19 \mathrm{ml}$ of Gadobenate dimeglumine (MultiHance ${ }^{\circledR}$, Bracco Diagnostics Inc., Monroe Township, N.J., USA).

\section{Statistical Analysis}

Unless otherwise stated, continuous variables are reported as mean \pm standard deviation or as median (interquartile range). Categorical variables are reported as proportions. Betweengroup comparisons for continuous variables were made with $t$ test and Mann-Whitney $U$ test, as appropriate. Categorical variables were compared using the $\chi^{2}$-test or Fisher's exact test, as appropriate.

We performed several multivariable logistic regression analyses to assess for factors independently associated with the presence of an abnormal MRV pelvis. For the primary analysis, baseline variables associated with an abnormal MRV pelvis in the univariable analysis (table $1, \mathrm{p}<0.2$ ), the presence of any predisposing risk factor as well as the presence of any condition associated with paradoxical embolism (table 2) were included in a multivariable logistic regression analysis. To avoid model overfitting we used a backward elimination method (likelihood ratio). Several additional analyses were then conducted. These analyses considered the following factors: (i) number of predisposing risk factors or (ii) history of miscarriages and cancer in lieu of any predisposing risk factor as well as (iii) number of conditions associated with paradoxical embolism or (iv) wake-up stroke in lieu of any condition associated with paradoxical embolism.

Variables that were retained in the each multivariable model were then included in a multivariable logistic regression analyses with bias-corrected, accelerated (BCa) 1000 resample bootstrap technique. Bootstrapping is a nonparametric approach to statisti- 
Table 1. Baseline characteristics as stratified by the presence of MRV pathology

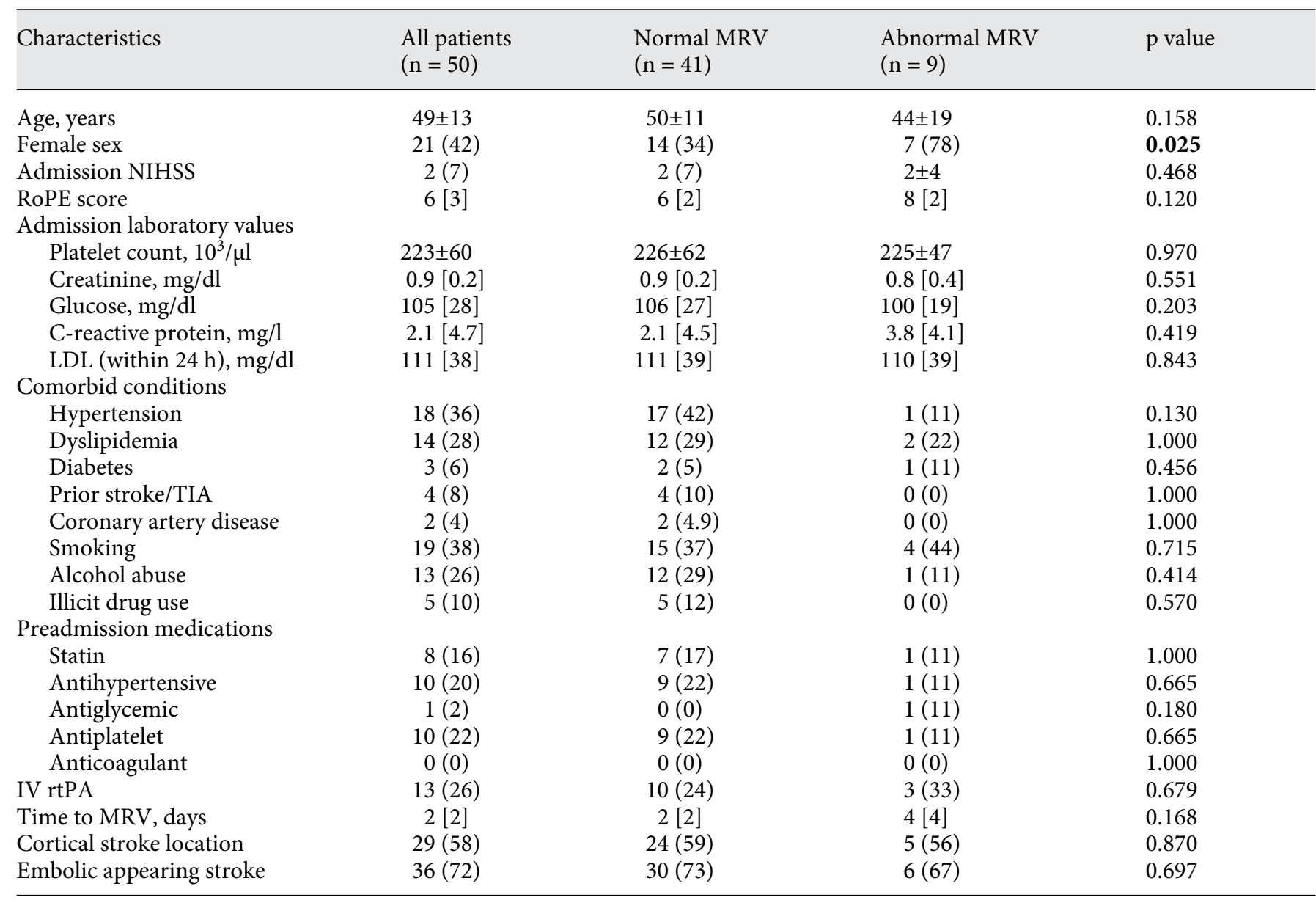

Data are $\mathrm{n}(\%)$, mean \pm standard deviation, and median [interquartile range]. IV $=$ Intravenous; LDL $=$ low-density lipoprotein cholesterol; NIHSS = National Institutes of Health Stroke Scale; MRV = magnetic resonance venography; RoPE = Risk of Paradoxical Embolism Study; rtPA = recombinant tissue-type plasminogen activator; TIA = transient ischemic attack.

cal inference that substitutes computations for more traditional distributional assumptions and asymptotic results. It is a method for deriving robust estimates of confidence intervals for estimates such as the odds ratio or regression coefficient. It has the advantage that it does not require distributional assumptions (except that it is assumed that the bootstrap sample is a random representation of the normally distributed population) and can provide more accurate inferences when the data are not well behaved or when the sample size is small. Therefore, it may become possible to estimate heretofore unknowable sampling distributions to provide secondary internal validation with more robust confidence intervals [16].

Collinearity diagnostics was performed and regression coefficients, their associated 95\% bootstrap confidence intervals, and $\mathrm{p}$ values were estimated for each model. The Hosmer-Lemeshow goodness-of-fit statistic was used to assess model fit. A two-sided $\mathrm{p}<0.05$ was considered statistically significant. All statistical analyses were performed using SPSS ${ }^{\circledR}$ Statistics 20.0.0 (IBM ${ }^{\circledR}$ Armonk, N.Y., USA).

\section{Results}

Figure 1 summarizes the flow chart of patient exclusion. Of the 50 patients studied, $9(18 \%)$ had an abnormal MRV. Of these, $4(8 \%)$ patients had a pelvic thrombus visualized by MRV and $5(10 \%)$ patients had a MayThurner anatomic variant, also known as iliocaval compression syndrome (fig. 2). All patients with pelvic DVT $(\mathrm{n}=4)$ were subsequently anticoagulated with warfarin. Of these, three had negative hypercoagulability testing (protein C, protein S, and antithrombin III; prothrombin G20210A mutation, Factor V Leiden, lupus anticoagulant, anticardiolipin antibody IgG and IgM, beta- 2 glycoprotein, and homocysteine) and one patient was lost to follow-up before the testing was done. Of note, when 
Table 2. Potential conditions as well as hypercoagulability and predisposing risk factors in patients with and without abnormal MRV

\begin{tabular}{|c|c|c|c|c|}
\hline Characteristics & $\begin{array}{l}\text { All patients } \\
(\mathrm{n}=50)\end{array}$ & $\begin{array}{l}\text { Normal MRV } \\
(\mathrm{n}=41)\end{array}$ & $\begin{array}{l}\text { Abnormal MRV } \\
(\mathrm{n}=9)\end{array}$ & $\mathrm{p}$ value \\
\hline \multicolumn{5}{|l|}{ Hypercoagulability factors $(\mathrm{HF})^{*}$} \\
\hline Protein $\mathrm{C}$ activity & $114(40)$ & $113(37)$ & $117(27)$ & 0.979 \\
\hline Protein S deficiency & $1(3)$ & $1(4)$ & $0(0)$ & 1.000 \\
\hline Protein $\mathrm{S}$ activity & $98 \pm 26$ & $101 \pm 28$ & $88 \pm 11$ & 0.256 \\
\hline AT III activity & $106(17)$ & $106(16)$ & $104(19)$ & 0.827 \\
\hline Homocysteine $>15 \mu \mathrm{mol} / \mathrm{l}$ & $2(10)$ & $2(13)$ & $0(0)$ & 1.000 \\
\hline Homocysteine, $\mu \mathrm{mol} / \mathrm{l}$ & $8(2)$ & $8(4)$ & $7(2)$ & 0.378 \\
\hline Prothrombin G20210A mutation & $0(0)$ & $0(0)$ & $0(0)$ & 1.000 \\
\hline Factor V Leiden mutation & $1(3)$ & $0(0)$ & $1(17)$ & 0.188 \\
\hline Lupus anticoagulant & $0(0)$ & $0(0)$ & $0(0)$ & 1.000 \\
\hline \multicolumn{5}{|l|}{ Risk factors predisposing to DVT (RF) } \\
\hline Oral contraceptive use & $3(6)$ & $2(0)$ & $1(11)$ & 0.456 \\
\hline History of cancer** & $4(8)$ & $2(5)$ & $2(22)$ & 0.144 \\
\hline History of DVT or PE & $1(2)$ & $1(2)$ & $0(0)$ & 1.000 \\
\hline History of miscarriages & $2(4)$ & $0(0)$ & $2(22)$ & 0.029 \\
\hline Recent travel & $2(4)$ & $1(2)$ & $1(11)$ & 0.331 \\
\hline Any predisposing RF present & $11(22)$ & $6(15)$ & $5(56)$ & 0.017 \\
\hline Number of predisposing RF & $0[0]$ & $0[0]$ & $1[1]$ & 0.013 \\
\hline \multicolumn{5}{|c|}{ Conditions associated with paradoxical embolism } \\
\hline Wake up stroke & $9(18)$ & $9(22)$ & $0(0)$ & 0.183 \\
\hline Valsalva maneuver & $5(10)$ & $4(10)$ & $1(11)$ & 1.000 \\
\hline Obstructive sleep apnea & $2(4)$ & $2(5)$ & $0(0)$ & 1.000 \\
\hline
\end{tabular}

Data are $\mathrm{n}(\%)$, mean \pm standard deviation, and median [interquartile range]. $*$ Percentages are based on the number of patients who had the test performed (respective imputing missing variables as normal or abnormal did not meaningfully change these associations; not shown). ${ }^{* *}$ All patients with a cancer history $(\mathrm{n}=4)$ were in remission (one patient with gastrointestinal cancer $>20$ years; one patient with small cell lung cancer and two patients with breast cancer $>5$ years). AT III = Antithrombin III; DVT $=$ deep vein thrombosis; $\mathrm{PE}=$ pulmonary embolism.

comparing rtPA-treated patients with and without abnormal MRV, there were no significant differences $(\mathrm{p}>$ 0.2 ) except that in the former group, all treated patients were females ( $\mathrm{p}=0.035 ; \mathrm{n}=2$ had May-Thurner syndrome and $\mathrm{n}=1$ had a pelvic DVT on subsequent MRV).

Table 1 summarizes the baseline characteristics of the studied population as stratified by the presence of MRV pathology. Overall, only female sex was associated with a higher risk of pelvic vein pathology (78 vs. $34 \%$; $p=$ 0.025). Otherwise, baseline characteristics were similar between patients with and without abnormal MRV pelvis. Though the RoPE score tended to be higher among patients with an abnormal MRV pelvis, there was no difference in the absolute score $(p=0.120)$ nor its distribution $(\mathrm{p}=0.560)$ as compared to patients with a normal MRV pelvis.

Table 2 summarizes the association between the abnormalities on the hypercoagulability profile, the presence of risk factors known to predispose to DVT, or the presence of conditions that have been associated with paradoxical embolism [13]. In summary, the presence of a condition potentially indicating paradoxical embolism was relatively high with $40 \%$ of patients having at least one potential condition identified. However, the preva- 
Fig. 1. Flowchart of patient exclusion.

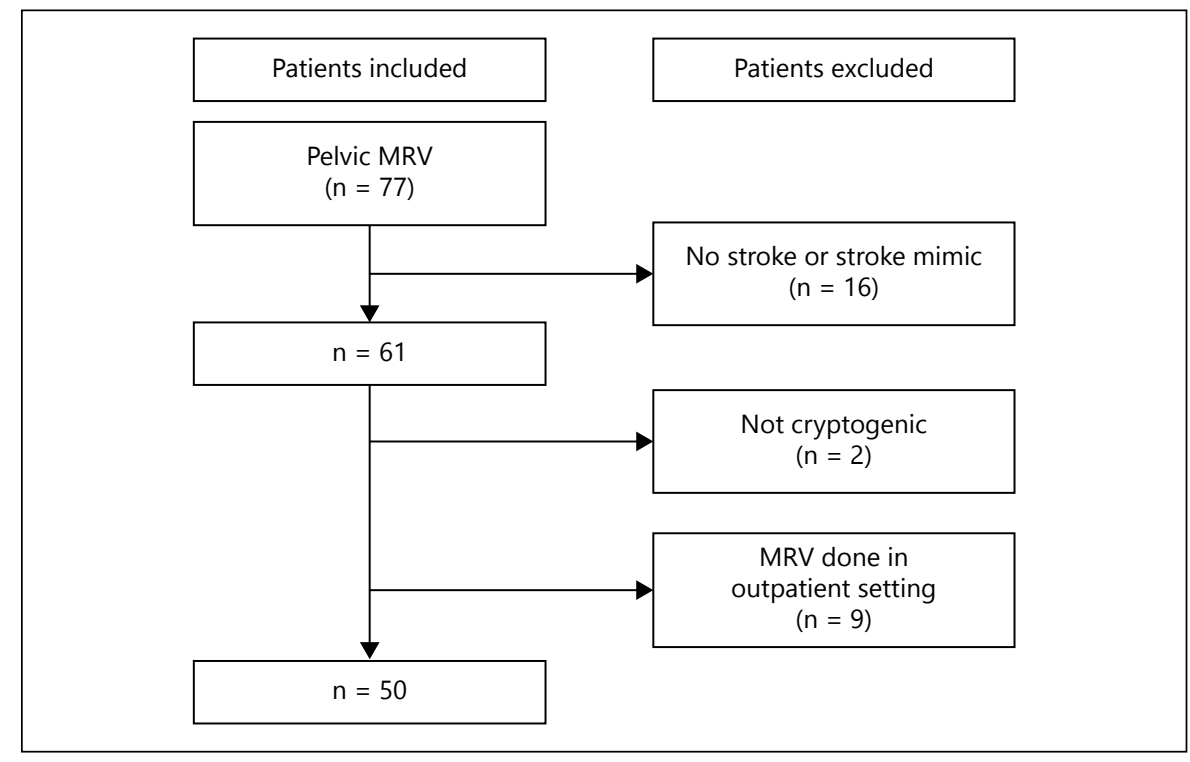

Fig. 2. MRV imaging. a, b Contrast enhanced T1 MRV indicates a hypointensity in the right internal iliac vein (white arrows; inset in b) with corresponding hyperintensity on non-enhanced $\mathrm{T} 1$ fat suppression MRV (inset in a, short arrow) consistent with thrombus. c, d Contrast enhanced T1 MRV shows narrowing of the left iliac vein (white arrows) caused by the overlying right iliac artery (asterisk, insets) consistent with May-Thurner anatomic variant.
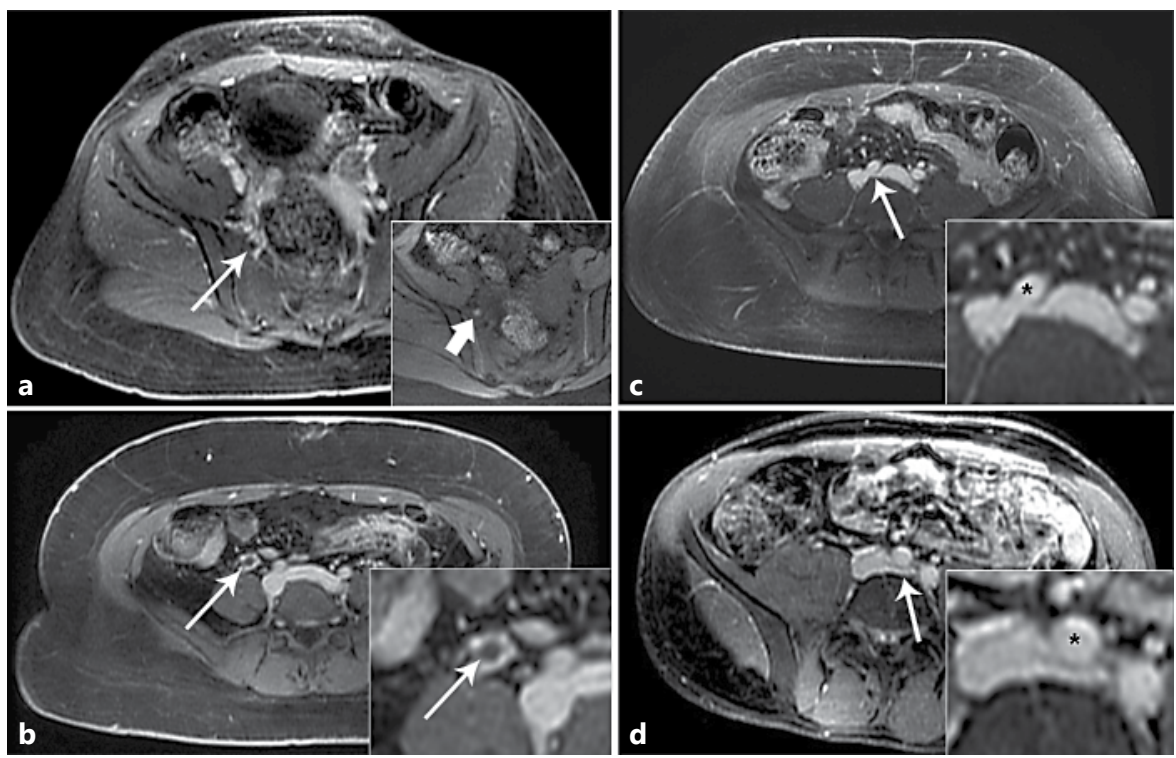

lence was similar between groups and neither the number (nor presence) of any such conditions was associated with an abnormal MRV pelvis ( $\mathrm{p}>0.1$ for all analyses). Further, there was no difference in the individual hypercoagulability factors between groups (assessed in $\mathrm{n}=29$ (71\%) and $8(89 \%)$ of patients with normal and abnormal MRV pelvis, respectively). Only one patient had more than one abnormal hypercoagulable factor (she had both protein $\mathrm{C}$ and protein $\mathrm{S}$ deficiency). Neither the number nor the presence of any abnormal hypercoagulability factor was associated with an abnormal MRV pelvis ( $\mathrm{p}>0.1$ for all analyses). Finally, a history of miscarriages ( $\mathrm{p}=$ $0.029)$, the presence of any risk factor known to predispose to DVT ( $p=0.017)$, and the number of predisposing risk factors $(\mathrm{p}=0.013)$ were significantly associated with the presence of pelvic vein pathology (table 2; fig. 3).

Multivariable logistic regression analyses confirmed that history of any predisposing risk factor (OR 6.7; coefficient 1.9; BCa 95\% CI 0.08-20.2; $\mathrm{p}=0.014$ ) as well as the number of predisposing risk factors (OR 3.9; coefficient 1.4; BCa 95\% CI 0.25-4.2; $\mathrm{p}=0.005$ ) were predictors of pelvic vein pathology on MRV (table 3 ). When predispos- 


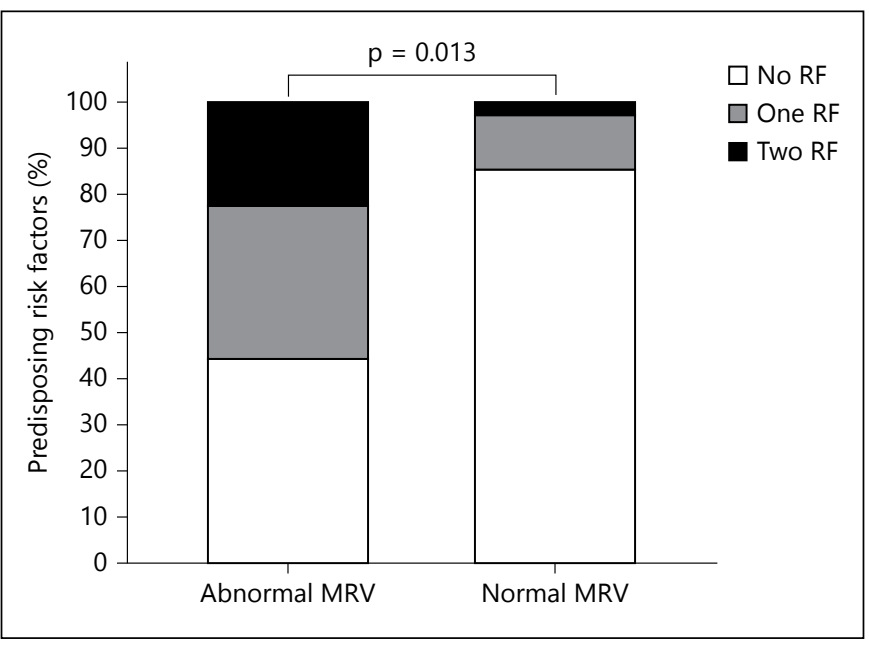

Fig. 3. Number of risk factors predisposing to DVT as stratified by pelvic vein pathology. Patients with an abnormal MRV pelvis had significantly more frequently a risk factor (RF) known to predispose to DVT than patients with a normal MRV pelvis ( $\chi^{2}$ test).

Table 3. Multivariable logistic regression analysis for factors associated with an abnormal MRV pelvis

\begin{tabular}{lll}
\hline Independent variable & $\begin{array}{l}\text { Regression } \\
\text { coefficient } \\
\text { (BCa 95\% CI)* }\end{array}$ & $\begin{array}{l}\mathrm{p} \\
\text { value }\end{array}$ \\
\hline Female sex & $1.4(-4.1$ to 23.0$)$ & 0.067 \\
$\begin{array}{l}\text { Number of predisposing risk factors* } \\
\text { Time to MRV pelvis (per day) }\end{array}$ & $\begin{array}{l}1.4(0.5 \text { to } 34.0) \\
0.2(-4.2 \text { to } 1.1)\end{array}$ & 0.029 \\
\hline
\end{tabular}

$\mathrm{BCa}=$ Bias corrected and accelerated; $\mathrm{CI}=$ confidence interval. Regression coefficients were based on 1,000 bootstrap samples. Hosmer-Lemeshow statistics: $\chi^{2} 5.120, p=0.529 .{ }^{*}$ Results were similar when entered as dichotomized (i.e., absent vs. present) variable (not shown).

ing risk factors were included individually, a history of miscarriages $(p=0.049)$ and remote cancer $(p=0.025)$ were independently associated with an abnormal MRV pelvis (not shown). Female sex ( $p>0.05)$ was not associated with pelvic vein pathology in any model (table 3 ). When we included the RoPE score in the primary multivariable logistic regression model instead of age and hypertension, it was not retained in the final model. Also, when forced back into the model it was not significantly predictive of an abnormal MRV pelvis (coefficient 0.194; BCa $95 \%$ CI -1.24 to 2.33 ; $\mathrm{p}=0.564$ ). Lastly, forcing the presence of any hypercoagulable abnormality and condition associated with paradoxical embolism into the mul- tivariable model confirmed that only the presence of DVT-predisposing risk factors predicted pelvic vein pathology in our population (OR 5.8; coefficient 1.7; BCa $95 \%$ CI 0.67-22.3; $\mathrm{p}=0.044)$.

\section{Discussion}

There are no published guidelines for the assessment of pelvic veins as part of the stroke workup and few studies have systematically investigated pelvic veins as a potential source for paradoxical emboli in CS patients $[6,10$, 12]. Moreover, data from the two larger studies have been conflicting with almost tenfold different prevalence rates (ranging from 2\% [12] to 20\% [6]). However, differences in the extent of diagnostic workup, classification of cryptogenic stroke, differences in imaging modalities and interpretation as well as different inclusion and exclusion criteria may partially account for this variability $[6,12]$.

In our study, the observed prevalence of pelvic DVT falls between these two extremes. The Paradoxical Emboli from Large Veins in Ischemic Stroke (PELVIS) study [6] reported 95 patients who underwent time-of-flight and non-contrast MRV. Compared to non-CS (i.e., stroke of determined etiology) patients, CS patients were younger, had a lower prevalence of vascular risk factors such as hypertension, smoking and prior history of stroke as well as more frequently had MRV with a high probability for pelvic DVT (20 vs. 4\%). However, only 7\% (3/40) of CS patients had an acute DVT, which is consistent with our reported prevalence of $8 \%$. In contrast, the more recent study by Liberman and colleagues [12] reported that $2.1 \%$ of CS patients with $\mathrm{PFO}$ had a pelvic DVT as determined by MRV. Patients with transient ischemic attack (i.e., no imaging evidence of cerebral ischemia) were included, which may have resulted in the misclassification of some patients and thus underestimation of DVT prevalence [12]. Conversely, the indication of a slightly higher prevalence in our study may relate to the fact that patients had their MRV performed at the treating physician's preference. This may have introduced bias to preferentially include patients where practitioners had a high index of suspicion for pelvic DVT and subsequent 'enrichment' of our sample with subjects more likely to have pelvic vein pathology. Indeed, we noted a relatively high prevalence of conditions suggesting paradoxical embolism [13], consistent with the previously noted prevalence in patients with a PFO [13]. This, and the fact that risk factors known to predispose to DVT were independent predictors for pelvic vein pathology on MRV in our study suggest that 
paradoxical embolism from pelvic DVT is implicated in the stroke pathogenesis of a significant subset of CS patients with a PFO. Thus, our observations indicate that pelvic MRV should be carefully considered in CS patients with PFO and negative lower extremity DUS if they have DVT risk factors. This is a particularly important observation because similar to prior observations [6], none of our patients with pelvic DVT had an abnormal thrombophilia profile. While not every patient in our sample had hypercoagulability testing, the overall observed frequency of abnormalities is consistent with prior observations [17]. Considering the significant costs associated with ordering an inherited thrombophilia workup, this highlights the need for judicious test selection and careful history taking with a focus on eliciting potential predisposing risk factors for DVT.

Unfortunately, we were unable to conduct a detailed comparison of CS patients with and without $\mathrm{PFO}$ that did vs. did not undergo MRV pelvis. However, we do not believe that this represents a major limitation but rather reflects 'real-life' practice and is thus of broad clinical importance. Nevertheless, further research is required to identify risk factors associated with pelvic vein pathology as a potential cause for CS in patients with PFO.

Our study expands on prior observations by presenting additional information regarding the prevalence of May-Thurner syndrome in our patient cohort. This variant was present in $10 \%$ of our sample adding to the notion that it may predispose to the development of venous thrombosis and paradoxical embolism [18]. Nevertheless, in the absence of visualized thrombosis, a causative relationship remains to be shown.

Strengths of our study relate to the investigation of pelvic vein pathology in a population of CS patients, with MRV in the acute post-stroke phase, who underwent extensive diagnostic evaluation. Importantly, we specifically sought to investigate CS patients with documented PFO and right to left shunt and negative lower extremity DUS. This is important because this represents the type of population where pelvic vein imaging is expected to be of highest yield and therefore has broad clinical applicability.

Several limitations of our study need to be considered. First, the retrospective study design and MRV assessment at the treating physician's discretion likely introduced bias and we cannot exclude potential confounding due to unknown or uncontrolled factors contributing to incident stroke. Since MRV pelvis was not systematically assessed in all CS patients, the true prevalence of pelvic pathology in CS patients with $\mathrm{PFO}$ could not be determined and further studies are warranted to confirm our findings and define patients most likely to benefit from pelvic vein imaging. Further, the sample size was relatively small. Though we utilized a bootstrapping approach for internal validation and more robust confidence intervals, this approach cannot overcome the inherent limitations associated with small sample sizes. Therefore, our results need to be interpreted cautiously and are considered important only for the sake of generating a hypothesis. Nonetheless, our sample expands upon current data, as it represents $>25 \%$ patients in published series systematically evaluating pelvic MRV in CS patients $[6,12]$. Our results indicate that MRV pelvis should be considered for CS workup in select patients, particularly if they have risk factors predisposing to DVT.

\section{Author Contributions}

Marcey Osgood: Data acquisition, critical revision of the manuscript for important intellectual content.

Elizabeth Budman: Data acquisition, critical revision of the manuscript for important intellectual content.

Raphael Carandang: Interpretation of data and drafting the article.

Richard P. Goddeau, Jr.: Critical revision of the manuscript for important intellectual content.

Nils Henninger: Study concept and design, data acquisition, statistical analysis, interpretation of data, and drafting the article.

\section{Sources of Funding}

This research received no specific grant from any funding agency in the public, commercial or not-for-profit sectors.

\section{Disclosure Statement}

The authors report no conflicts of interest.

References

1 Sacco RL, Ellenberg JH, Mohr JP, Tatemichi TK, Hier DB, Price TR, Wolf PA: Infarcts of undetermined cause: the NINCDS stroke data bank. Ann Neurol 1989;25:382-390.

2 Cabanes L, Mas JL, Cohen A, Amarenco P, Cabanes PA, Oubary P, Chedru F, Guerin F, Bousser MG, de Recondo J: Atrial septal aneurysm and patent foramen ovale as risk factors for cryptogenic stroke in patients less than 55 years of age. A study using transesophageal echocardiography. Stroke 1993;24:1865-1873.

3 Steiner MM, Di Tullio MR, Rundek T, Gan R, Chen X, Liguori C, Brainin M, Homma S, Sacco RL: Patent foramen ovale size and embolic brain imaging findings among patients with ischemic stroke. Stroke 1998;29:944-948. 
4 Putaala J, Tatlisumak T: Prime time for dissecting the entity of cryptogenic stroke. Stroke 2014;45:950-952.

5 Gautier JC, Durr A, Koussa S, Lascault G, Grosgogeat Y: Paradoxical cerebral embolism with a patent foramen ovale. Cerebrovasc Dis 1991;1:193-202.

6 Cramer SC, Rordorf G, Maki JH, Kramer LA, Grotta JC, Burgin WS, Hinchey JA, Benesch C, Furie KL, Lutsep HL, Kelly E, Longstreth WT Jr: Increased pelvic vein thrombi in cryptogenic stroke: results of the paradoxical emboli from large veins in ischemic stroke (PELVIS) study. Stroke 2004;35:46-50.

7 Ranoux D, Cohen A, Cabanes L, Amarenco P, Bousser MG, Mas JL: Patent foramen ovale: is stroke due to paradoxical embolism? Stroke 1993;24:31-34.

8 Lethen H, Flachskampf FA, Schneider R, Sliwka U, Kohn G, Noth J, Hanrath P: Frequency of deep vein thrombosis in patients with patent foramen ovale and ischemic stroke or transient ischemic attack. Am J Cardiol 1997; 80:1066-1069.
9 Stollberger C, Schneider B, Abzieher F, Wollner T, Meinertz T, Slany J: Diagnosis of patent foramen ovale by transesophageal contrast echocardiography. Am J Cardiol 1993;71:604-606.

10 Cramer SC, Maki JH, Waitches GM, D'Souza N, Grotta JC, Burgin WS, Kramer LA: Paradoxical emboli from calf and pelvic veins in cryptogenic stroke. J Neuroimaging 2003;13: 218-223.

11 Cramer SC, Rordorf G, Kaufman JA, Buonanno F, Koroshetz WJ, Schwamm L: Clinically occult pelvic-vein thrombosis in cryptogenic stroke. Lancet 1998;351:1927-1928.

12 Liberman AL, Daruwalla VJ, Collins JD, Maas MB, Botelho MP, Ayache JB, Carr J, Ruff I, Bernstein RA, Alberts MJ, Prabhakaran S: Diagnostic yield of pelvic magnetic resonance venography in patients with cryptogenic stroke and patent foramen ovale. Stroke 2014; 45:2324-2329.

13 Ozdemir AO, Tamayo A, Munoz C, Dias B, Spence JD: Cryptogenic stroke and patent foramen ovale: clinical clues to paradoxical embolism. J Neurol Sci 2008;275:121-127.

14 Adams HP Jr, Bendixen BH, Kappelle LJ, Biller J, Love BB, Gordon DL, Marsh EE 3rd: Classification of subtype of acute ischemic stroke. Definitions for use in a multicenter clinical trial. TOAST. Trial of Org 10172 in Acute Stroke Treatment. Stroke 1993;24:35-41.
15 Kent DM, Ruthazer R, Weimar C, Mas JL, Serena J, Homma S, Di Angelantonio E, Di Tullio MR, Lutz JS, Elkind MS, Griffith J, Jaigobin C, Mattle HP, Michel P, Mono ML, Nedeltchev K, Papetti F, Thaler DE: An index to identify stroke-related vs incidental patent foramen ovale in cryptogenic stroke. Neurology 2013;81:619-625.

16 Steyerberg EW, Harrell FE Jr, Borsboom GJ, Eijkemans MJ, Vergouwe Y, Habbema JD: Internal validation of predictive models: efficiency of some procedures for logistic regression analysis. J Clin Epidemiol 2001;54:774781.

17 Morris JG, Singh S, Fisher M: Testing for inherited thrombophilias in arterial stroke: can it cause more harm than good? Stroke 2010; 41:2985-2990.

18 Greer DM, Buonanno FS: Cerebral infarction in conjunction with patent foramen ovale and May-Thurner syndrome. J Neuroimaging 2001;11:432-434. 